Regular Article

\title{
The effect of sharp solid edges on the droplet wettability
}

\author{
Zhanlong Wang, Kui Lin, Ya-Pu Zhao*
}

State Key Laboratory of Nonlinear Mechanics, Institute of Mechanics, Chinese Academy of Sciences, Beijing 100190, China School of Engineering Science, University of Chinese Academy of Sciences, Beijing 100049, China

\section{G R A P H I C A L A B S T R A C T}

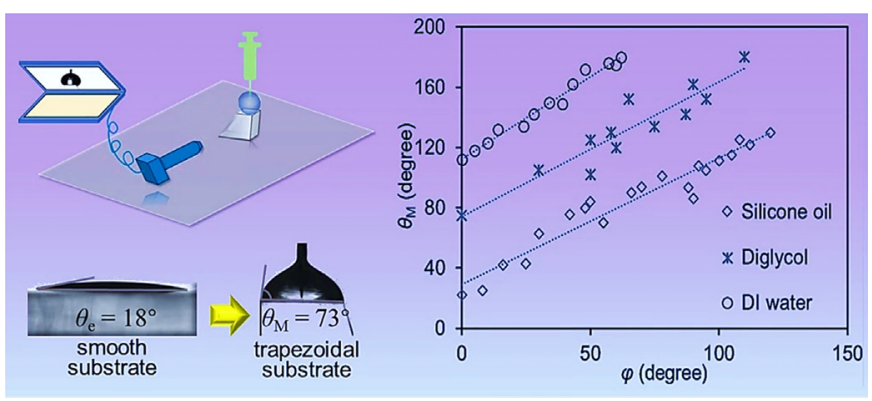

\section{A R T I C L E I N F O}

\section{Article history:}

Received 7 March 2019

Revised 23 May 2019

Accepted 24 May 2019

Available online 25 May 2019

\section{Keywords:}

Droplet wettability

Contact angle

Sharp edge

Contact line pinning

\begin{abstract}
A B S T R A C T
Understanding the role of pinning force in droplet dynamic wetting is of critical importance for surface science studies. Generally, the pinning force is only related to the surface tension and the change of contact angle. However, there is an obvious correlation between the pinning force and the surface geometry. In this paper, the relation between the surface geometry and contact line pinning was studied with systematical experiments and theoretical analysis. We compared the samples with different edge angles and carried out plenty experiments with different liquids. Meanwhile, the theoretical analysis and molecular simulation were carried out. The results show that the sharp edge has a strong pinning effect on the contact line and can significantly change the contact angle and wetting state of droplets. The maximum contact angle of droplet has a linear relation with the edge angle of substrate. The formula of pinning force was revised to consider the impact of surface topography. According to the relationship between surface defect and contact line, we proposed a model to classify the cases of contact line pinning for the first time. Our research will deepen the understanding of contact line pinning and provide help for potentially industrial production designs.
\end{abstract}

(c) 2019 Elsevier Inc. All rights reserved.

\section{Introduction}

Droplet wetting on a solid surface is ubiquitous, and it is applied in numerous fields, e.g., super-hydrophobic surfaces [1-3], printing [4], self-cleaning surfaces [5,6], water harvesting [7,8], heat exchangers [9-11], self-assembly [12,13] and microfluidics [14].

\footnotetext{
* Corresponding author at: State Key Laboratory of Nonlinear Mechanics (LNM), Institute of Mechanics, Chinese Academy of Sciences, No. 15, Beisihuanxi Road, Beijing 100190, China.

E-mail address: yzhao@imech.ac.cn (Y.-P. Zhao).
}

Natural solid surfaces or artificial surfaces $[15,16]$ are rough generally, and the liquid spreading on these surfaces is often affected by the surface defects. During the droplet spreading, when the contact line encounters these defects, the contact line will be pinned. The force that prevents the contact line from moving is called the pinning force $[17,18]$. On specifically designed surfaces, the pinning force enables the ability to control droplet movement [19], particle self-assembly [13] and various deposit patterns formation [20-22]. Hindered by pillars of solid surfaces, a receding contact line exhibits the shape of a logarithmic function [23], and the contact line depends on the defect microstructure. The elastic restoring force 
follows Hooke's law and is dependent on the properties of the fluid and contact angle on the underlying surface [24].

For a droplet on a solid surface, its wetting state is controlled by liquid-vapor and solid-vapor surface tension, and the liquid-solid interface tension $[25,26]$. On rough surfaces, wetting is not only affected by surface tension, but surface imperfections also play an important role in droplet spreading. This is due to the pinning of moving contact line by the surface defects [23]. The pinning force is crucial in changing the droplet's wetting state $[18,27,28]$. It usually originates from the roughness of the surface and expressed as:

$F_{\mathrm{p}}=\gamma_{\mathrm{lv}}\left(\cos \theta-\cos \theta_{\mathrm{e}}\right)$

where $\gamma_{\mathrm{lv}}$ is the liquid-vapor surface tension, $\theta$ is the real-time contact angle, $\theta_{\mathrm{e}}$ is the equilibrium contact angle (ECA). In this formula, the pinning force is only related to the changes of surface tension and contact angle, and the influence of surface geometry on the pinning force is not considered. Mason et al. mentioned that the sharp edge significantly hinders the movement of contact line and curved smooth surfaces have better wetting and dewetting properties than surfaces with sharp edges [29]. Hensel et al. compared the hydrophobic properties of surfaces covered by tiny mushroom and serif T structures and the results indicated the decisive role of the sectional profile of overhanging structures to form a robust heterogeneous wetting state for low-surface-tension liquids that enables the omniphobicity [30]. De Wijs et al. employed a modified Wilhelmy plate testing technique and measured the force for meniscus shape transitions over edge geometries under quasi-static conditions. Moreover, wetting effects directly linked to surface defects can be detected qualitatively [31]. All these studies show that the pinning of the contact line shows obvious geometric dependence [29-34]. As a further illustration, as depicted in Fig. 1, a diglycol droplet is deposited on a Polydimethylsiloxane (PDMS) solid block with four edge angles (EAs) of $60^{\circ}, 80^{\circ}, 90^{\circ}$ and $90^{\circ}$, respectively. The ECA of diglycol droplet is $70 \pm 3^{\circ}$ and the advancing contact angle (ACA) is $80 \pm 3^{\circ}$. The maximum contact angle (MCA) of the droplet exceeds $150^{\circ}$. This also indicates that the sharp edge has a strong pinning effect on the movement of moving contact line can significantly alter the wetting state of the droplets. This phenomenon can be seen everywhere in daily life, such as the process of watering flowers, etc. Despite the numerous examples in life and lots of research has been done on this, how the droplet wetting state changes with the sharp edge and how the pinning force is related to geometric factors has not been well solved. Pinning force originates from surface defect or discontinuity of surface properties, even for the weak nanoscale defects can be the source of a plethora of hysteretical phenomena [35,36]. The studies carried out by Wang et al. [35] and Giacomello et al. [36] revealed that the pinning force originates from a nanoscopic effects. Chan et al. [37] reported the fast dynamics of a three phase contact line moving over surface nanobubbles, polymeric droplets, and hydrophobic particles, and found the fast receding and pinning-depinning processes for polymeric droplets and solid particles, respectively. Fetzer et al. [38] studied the advancing and receding water contact angles on tailored rough surfaces, and found the asymmetries in terms of contact angle values and the shape of the contact area. They attributed these discrepancies to the contact line curvature and different pinning forces, which reflecting the surface geometry. Kalinin et al. [39] indicated that the microscale topography of a solid surface affects the apparent advancing and receding angles at the contact line of a liquid drop pinned to this surface. Perrin et al. [40] also points out that the defects even at the atomic scale affect the contact line motion. So, how the sharp edges of different edge angles affect the wetting process at atomic scale? Defects of different densities will lead to different contact line shapes and hence different pinning strength [41]. Whether can we classify the situation of the contact line pinning according to the defect density?

In this paper, we carried out a detailed experimental study comparing the effects of different angular sharp edge on the wetting process of liquid. The relation between the droplet wetting state and the edge geometry angle was given. The expression formula of pinning force was revised. The high energy barrier for liquid molecules to cross the sharp edge was observed from the molecular point of view. In addition, the cases of contact line pinning were classified into three models according the relation of defects and contact line. Our research may deepen the understanding of the
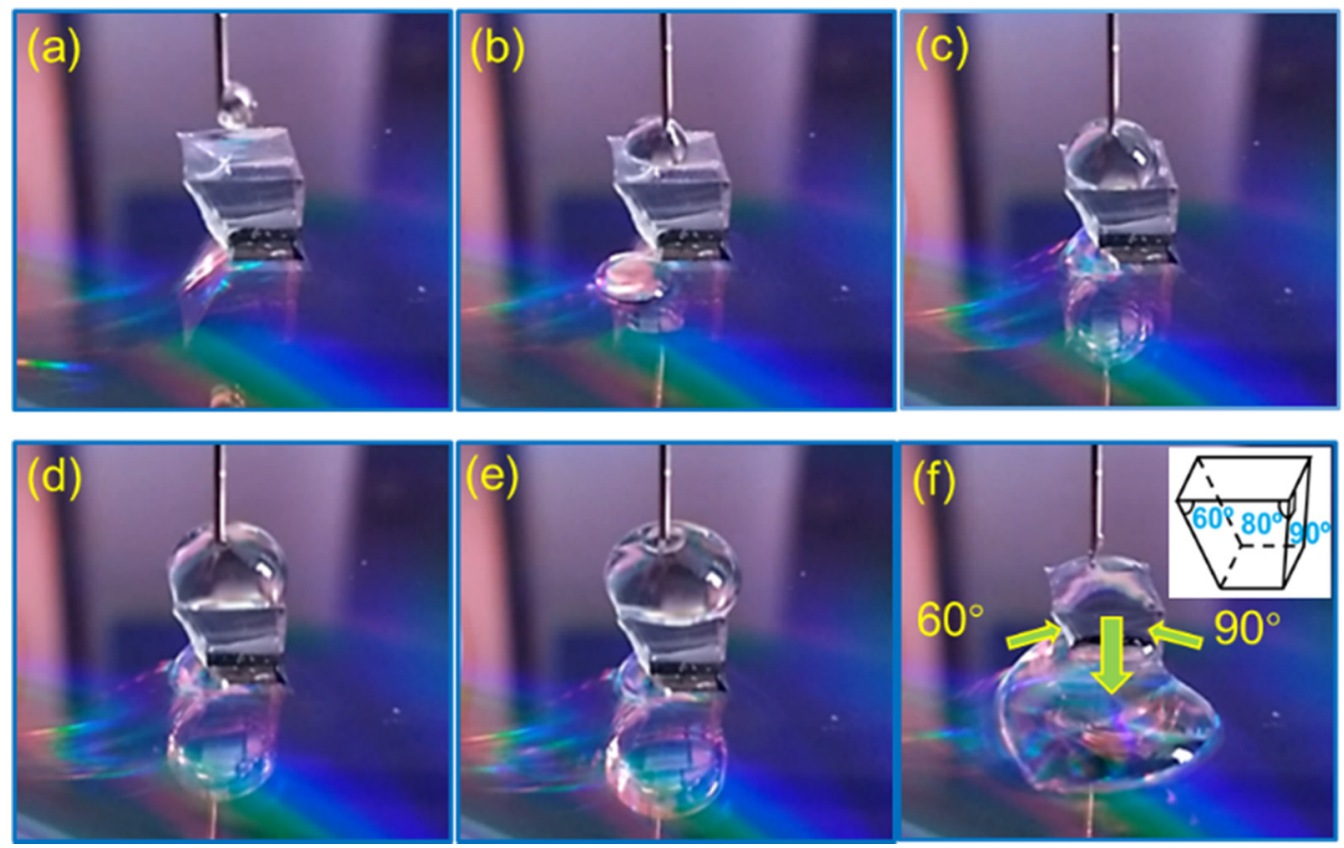

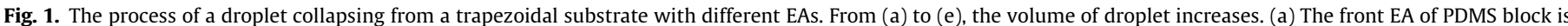

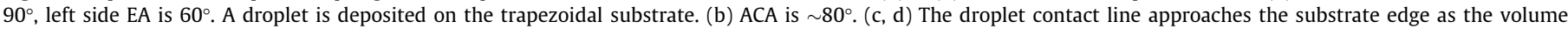
increases. (e) The contact angle approaches $180^{\circ}$. (f) The droplet collapses over the side where the EA is equal to $90^{\circ}$. Insert: the illustration of the EAs of the substrate. 
pinning effect in droplet wetting, and contribute to the design of super lyophobic or droplet movement-controlling surfaces, etc. in the future $[3,13,19]$.

\section{Materials and methods}

\subsection{Materials}

The substrates used in experiments are made of PDMS (Sylgard 184, Dow Corning). The solid blocks of PDMS are fabricated in two steps: (i) Mixing PDMS with resin and a curing agent (Dow Chemical Co.) at a ratio of 10:1 by mass. The PDMS is then made into several small cuboids, $0.5 \times 2 \times 5 \mathrm{~cm}^{3}$ in size, and solidified at $80^{\circ} \mathrm{C}$ for $4 \mathrm{~h}$; (ii) Cutting the cuboids into trapezoids, with different EAs on each of the four faces. The elastic modulus of the PDMS used in this paper is larger than $3 \mathrm{MPa}$ [42]. The true advancing and receding contact angle can be calculated with the following formulas [43]:

$\theta_{a} \approx \bar{\theta}_{a}+\frac{6 \gamma \sin \theta_{a}}{b E}$

$\theta_{r} \approx \bar{\theta}_{r}+\frac{6 \gamma \sin \theta_{r}}{b E}$

where $\theta_{a}, \theta_{r}, \bar{\theta}_{a}$ and $\bar{\theta}_{r}$ are the observed advancing, recending contact angle on soft substrates, and the true advancing, receding contact angle on polymers without the surface deformation, respectively. $\gamma, E$ and $b$ are the surface tension of liquid, elastic modulus and a typical length scale of micron order that remained unspecified. According to the equations above, we can get that $\theta_{a} \approx \bar{\theta}_{a}$ and $\theta_{r} \approx \bar{\theta}_{r}$. Therefore, the influence of substrate softness on contact angle can be neglected.

In our experiments, silicone oil, diglycol and deionized water (DI water) are employed. The properties of these liquids are listed in Table 1 . The droplets of DI water and diglycol partially wets the substrate with an ECA of $\sim 110 \pm 3^{\circ}$ and $\sim 75 \pm 3^{\circ}$, respectively. The droplet of silicone oil totally wets the substrate surface.

The droplet contact angle is captured by a contact angle measurement system (OCA 20, Dataphysics). The volume of the droplet during the experiments is controlled by the injection system of the OCA 20. We control the injection velocity to ensure the stable growth of the droplet. The schematic diagrams of PDMS trapezoidal substrate and measurement setup are shown in Fig. 2.

\subsection{Schematic diagram of edge effect}

The interaction of droplet and substrate edge contains the cases that a droplet spreads uphill and downhill (Fig. 3). During the droplet spreading, the sharp edge will exert an additional force $F_{\mathrm{a}}$ on the contact line. In the downhill spreading, the additional force acting as pinning force $F_{\mathrm{p}}$ points to the reverse direction of droplet spreading. Therefore, the additional force prevents the droplet from spreading (Fig. 3a and b). At the moving contact line, the force balance is expressed as $\gamma_{\mathrm{sl}}+F_{\mathrm{p}}-\gamma_{\mathrm{sv}}-\gamma_{\mathrm{lv}} \cos \theta=0$, where $\gamma_{\mathrm{lv}}, \gamma_{\mathrm{sl}}$ and $\gamma_{\mathrm{sv}}$ are the surface tension of liquid-vapor, solid-liquid and solidvapor, respectively. In Fig. 3, $\theta$ is the real-time contact angle of droplet, and $\varphi$ is the EA of substrate. In another case, the additional force will draw the droplet uphill, and contribute to the droplet spreading.

Table 1

The properties of liquids used in experiments.

\begin{tabular}{llll}
\hline Liquid $(298 \mathrm{~K})$ & $\begin{array}{l}\text { Surface tension } \\
(\mathrm{mN} / \mathrm{m})\end{array}$ & $\begin{array}{l}\text { Density } \\
\left(\mathrm{g} / \mathrm{cm}^{3}\right)\end{array}$ & $\begin{array}{l}\text { Viscosity } \\
(\mathrm{mPa} \mathrm{s})\end{array}$ \\
\hline Silicone oil & 20.9 & 0.963 & 50.0 \\
Diglycol & 42.1 & 1.118 & 30.0 \\
DI water & 72.0 & 1.000 & 0.895 \\
Ethanol & 21.97 & 0.789 & 1.074 \\
\hline
\end{tabular}
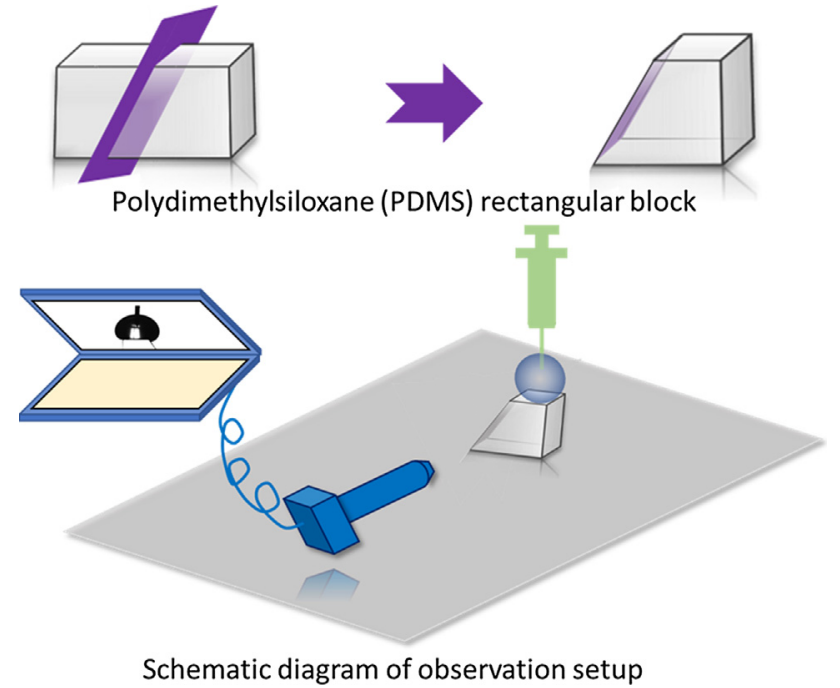

Fig. 2. The schematic diagram of PDMS trapezoidal substrate and the schematic diagram of measurement setup.

The additional force acts as an attraction and the forces balance as $\gamma_{\mathrm{sl}}-F_{\mathrm{a}}-\gamma_{\mathrm{sv}}-\gamma_{\mathrm{lv}} \cos \theta=0$ (Fig. $3 \mathrm{c}$ and $\mathrm{d}$ ). Here, we mainly focus on the case where droplet spreads downhill, i.e., the case that the pinning force prevents the droplet from spreading. Droplets stay on the solid surface in two states: (I) the droplet stays totally on the horizontal surface, (II) the droplet crosses the substrate edge and some liquid occupies the slope side (Fig. 3e). In different cases, the force balance at the contact line varies. In Case I, $\gamma_{\mathrm{sl}}$ and $\gamma_{\mathrm{sv}}$ work along the horizontal surface, and $\gamma_{\mathrm{lv}}$ works along the droplet surface. In Case II, $\gamma_{\mathrm{sl}}$ and $\gamma_{\mathrm{sv}}$ work along the slope surface (Fig. 3f). In Fig. 3g, the diagrams of ECA, ACA and MCA are also given. As the contact line approaches the substrate edge, the droplet maintains the ACA until the contact line reaches the edge, and after that, the droplet contact angle changes from the ACA to the MCA before the droplet collapses from the horizontal surface to the slope surface $[30,44]$.

In droplet wetting, two dimensionless numbers must be considered, the Capillary number and the Bond number, which represent the competition between viscosity and surface tension, gravity and surface tension, respectively. The Capillary number is defined as $C a=\mu v / \gamma$, and the Bond number is defined as $B o=\rho g r^{2} / \gamma$, where $\mu, v, \rho, g, r$ are viscosity, velocity of droplet movement, density, gravitational acceleration and droplet radius, respectively. The capillary numbers are of $C a \approx 10^{-4} \cdots 10^{-3} \ll 1$ for the liquids used in experiments, and the effect of liquid viscosity on droplet contact angle can be ignored. In the experiments, as the liquid is injected into the droplet, the Bond number becomes larger. However, the droplets will retain their constant ACAs as the Bond number increases $[45,46]$. Therefore, we can regard the effect of gravity on the experimental results to be small.

\section{Theoretically analysis}

The contact line is usually pinned when it meets an edge with the spreading of a droplet on a rough surface. The pinning force arises as the contact angle increases. In previous works $[18,27,28]$, the pinning force can be expressed by Eq. (1). When the contact line is pinned by the edge on horizontal surface, the pinning force as the contact angle changes from ACA to MCA can be expressed as:

$F_{\mathrm{p}}=\gamma_{\mathrm{lv}}\left(\cos \theta_{\mathrm{a}}-\cos \theta_{\mathrm{M}}\right)$

where $\theta_{\mathrm{a}}$ is the ACA, and $\theta_{\mathrm{M}}$ is the MCA. When the droplet overcomes the barrier, the contact line will pass over the sharp edge, and the 
(a)

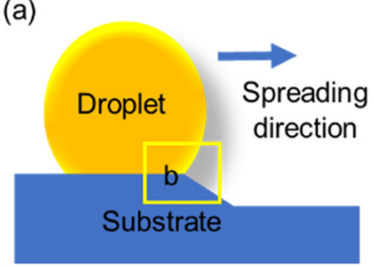

(c)

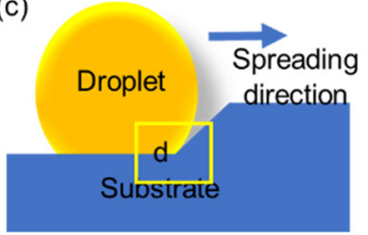

(b)

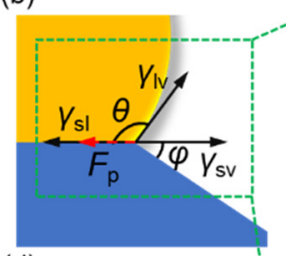

(d)

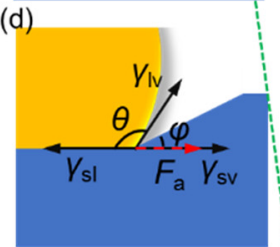

(e)
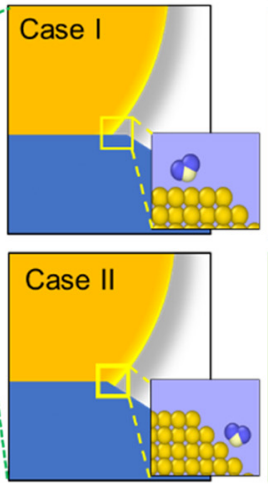

(f)
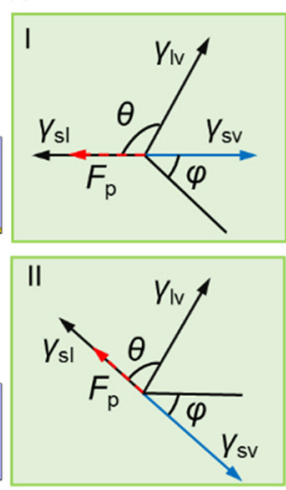

(g)

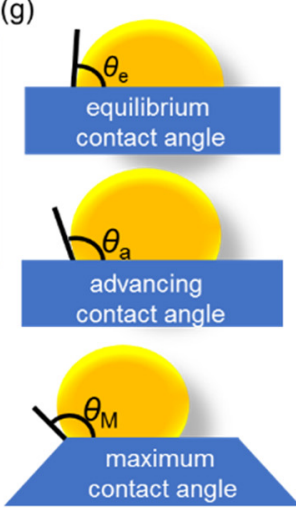

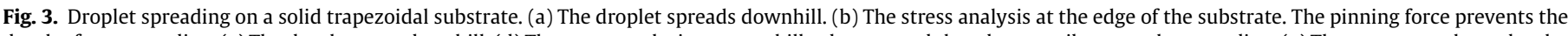

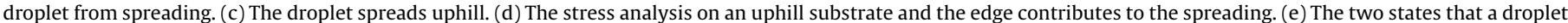

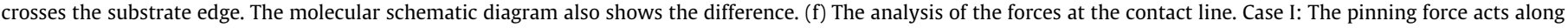
the horizontal plane. Case II: The pinning force points to the direction of slope plane. (g) The diagram of ECA, ACA and MCA.

contact angle between the droplet and the inclined surface will become $\theta_{\mathrm{M}}-\varphi$. The contact angle changes from $\theta_{\mathrm{M}}$ to $\theta_{\mathrm{M}}-\varphi$. Since the displacement of the contact line is assumed to be infinitesimally small, only from the horizontal plane across the edge to the inclined plane, it can be assumed that the contact line does not move. The change of free energy is only related to the change of gas-liquid interface contact angle, i.e $\Delta E=\gamma_{\mathrm{lv}}\left[\cos \left(\theta_{\mathrm{M}}-\varphi\right)-\cos \theta_{\mathrm{M}}\right] \mathrm{d} A$. Thus, the force related to the released energy per unit length is:

$F_{\mathrm{s}}=\gamma_{\mathrm{lv}}\left[\cos \left(\theta_{\mathrm{M}}-\varphi\right)-\cos \theta_{\mathrm{M}}\right]$

The pinning force before the droplet crosses the sharp edge is the same as the force needed to cross the overcome the energy barrier, namely:

$F_{\mathrm{p}}=F_{\mathrm{s}}$

Thus, we can get the relationship between the contact angle and the EA:

$\cos \theta_{\mathrm{a}}-\cos \theta_{\mathrm{M}}=\cos \left(\theta_{\mathrm{M}}-\varphi\right)-\cos \theta_{\mathrm{M}}$

namely:

$\cos \theta_{\mathrm{a}}=\cos \left(\theta_{\mathrm{M}}-\varphi\right)$

The relation can then be expressed as:

$\theta_{\mathrm{M}}=\theta_{\mathrm{a}}+\varphi$

or

$\theta_{\mathrm{M}}=\theta_{\mathrm{e}}+\varphi+\frac{1}{2} \theta_{\mathrm{h}}$

where $\theta_{\mathrm{h}}$ is the contact angle hysteresis. $\theta_{\mathrm{M}}$ is not larger than $180^{\circ}$. For different materials, the slope of $\theta_{\mathrm{M}}$ with respect to $\varphi$ is different. Here, we introduce a parameter $\varsigma$ to show this difference. So the pinning force of contact angle changing from ECA to MCA is expressed as:

$F=\gamma_{\mathrm{lv}}\left[\cos \theta_{\mathrm{e}}-\cos \left(\theta_{\mathrm{a}}+\varsigma \varphi\right)\right]$

where $\varsigma$ is the slope of contact angle with edge angle, and it depends on the properties of materials. The energy released as the contact line travels from the horizontal surface to the slope surface can be expressed as:

$\Delta E=\gamma_{\mathrm{lv}}\left[\cos \left(\theta_{\mathrm{M}}-\varphi\right)-\cos \theta_{\mathrm{M}}\right] \mathrm{d} A$

where $\mathrm{d} A$ is the area of the increased droplet-solid interface. The contact line was pinned at the solid edge, and the energy increases as the droplet volume increases. The contact line moves from the horizontal surface to the slope surface when droplet collapses. Fig. 4 shows the theoretical results of the variation of the MCA and the energy barrier with EA.
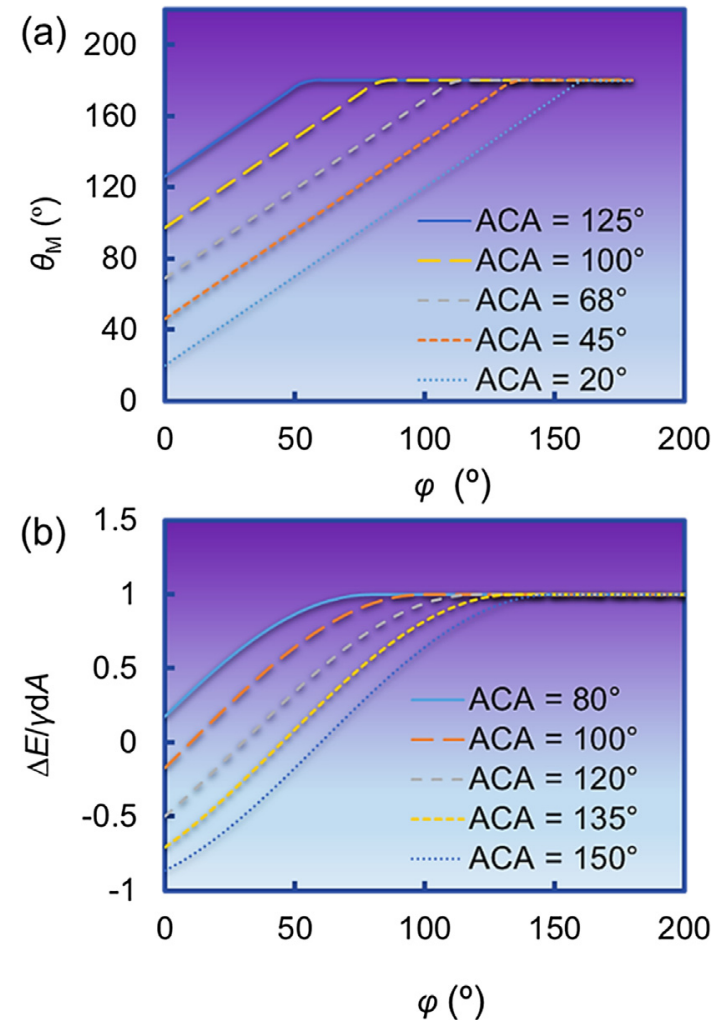

Fig. 4. The theoretical analysis MCA and energy barrier versus EA. (a) Variation of the MCA against different EAs. The MCA increases linearly with EA before reaching $180^{\circ}$. (b) The variation of energy barriers with EA. The energy barrier increases as the EA increases. When the edge angle is fixed, the energy barrier increases with ACA for different liquids.

\section{Results and discussions}

\subsection{Theoretical analysis of the MCA with varying EAs}

Fig. 4 shows the theoretical prediction of the MCA versus the EA. The theoretical analysis shows that MCA varies with EA linearly (Fig. 4a). For substrates with different EAs, the variation between the MCA and EA follows the same law. We assume that the contact angle is no larger than $180^{\circ}$. In Fig. 4(b), the free energy difference (FED) before and after the contact line crosses the edge is shown theoretically. The theoretical analysis of free energy shows that FED is related to the EA by a cosine function. For most liquids, as 
the EA increases, FED increases. When the EA is fixed, the FED is different for different liquids.

\subsection{The collapse of droplet and the MCA that the substrate can support}

When a droplet is placed on an infinitely smooth surface, the droplet will continue to advance forward with a constant ACA as the liquid continues to be injected into the droplet. However, when the droplet is placed on a bounded substrate, the contact line of the droplet will be pinned by the edge when the contact line meets the edge. And the spreading of droplet will be impeded. As the contact line is pinned, the droplet will have a contact angle greater than the ACA with the continuous injection of liquid. Fig. 5 shows the edge effect on the wetting state of droplets (also see Movies S1, S2 and S3). In Fig. 5(a) and (b), as the droplet grows, it maintains a constant contact angle on the infinite surface, and presents an increasing contact angle on the trapezoidal finite boundary substrate. For different liquids, the droplets obey the same rule. On these trapezoidal substrates, different liquids will have different MCAs, and this is due to the properties of liquids and substrates.

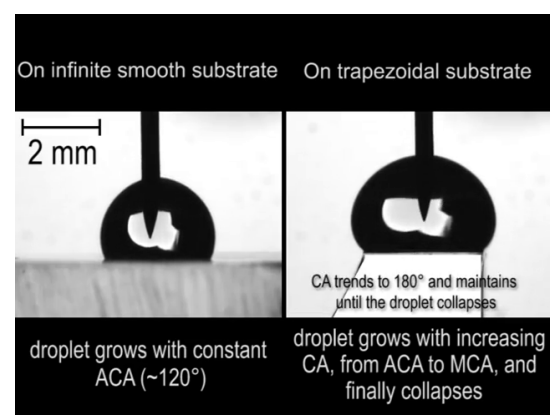

Movie S1.

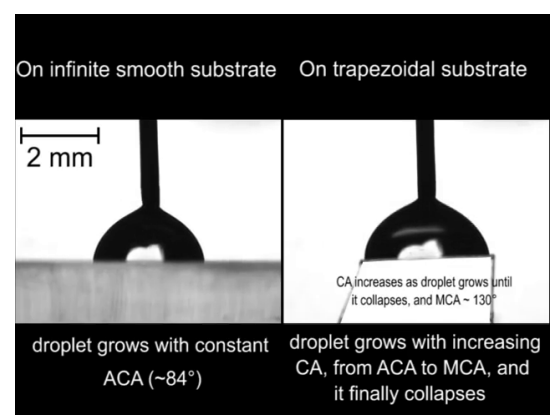

Movie S2.

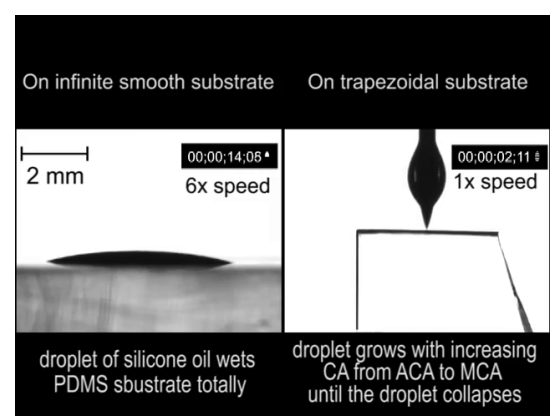

Movie S3.
Here, the droplet of diglycol is used to illustrate this. As shown in Fig. 6(a), the ECA of the droplet is $75 \pm 3^{\circ}$ (high), the ACA is $80 \pm 3^{\circ}$ and the receding contact angle is $70 \pm 3^{\circ}$. The contact angle hysteresis is $\sim 10^{\circ}$. For illustration, we compare two cases of trapezoidal substrates with the obtuse (Fig. 6b) and acute angles (Fig. 6c) on the right side, respectively. The EAs of the two substrates are $\varphi=90 \pm 3^{\circ}, 110 \pm 3^{\circ}$ (Fig. 6b) and $\varphi=90 \pm 3^{\circ}, 60 \pm 3^{\circ}$ (Fig. 6c), respectively. When the liquid is continuously injected into the droplet, the contact angle of the droplet becomes larger and larger. As the droplet grows, the contact angle reaches $180^{\circ}$ (Fig. $6 \mathrm{~b}$ and c; middle). When the volume of the droplet exceeds the critical value, the droplet collapses from the side of the EA $\varphi=90 \pm 3^{\circ}$ (Fig. 6b) and $\varphi=60 \pm 3^{\circ}$ (Fig. 6c), respectively. This phenomenon occurs for all the liquids used in experiments. In Fig. 6 (d), the profile details of droplets near the substrate edge is shown. As the droplet becomes larger, the profile gradually flattens. The contact angle approaches $180^{\circ}$ as the contact angle changes from $\theta_{V 1}$ to $\theta_{V 3}$.

\subsection{The relation between MCA and substrate EA}

In Fig. 7, we compared the growth and collapse of droplets on substrates with different EAs. In Fig. 7(a), the EAs of these substrates are $46 \pm 3^{\circ}, 68 \pm 3^{\circ}$ and $77 \pm 3^{\circ}$, respectively. From the left to the right, the figure shows the process of droplet dropping and spreading successively, including: droplet dropping on the surface of the substrate, droplet spreading with ACA, contact line being pinned, and droplet collapses from horizontal surface after overcoming the pinning force. On these three substrates, the MCAs are $82 \pm 3^{\circ}, 95 \pm 3^{\circ}$ and $103 \pm 3^{\circ}$, respectively (Movie S4). The cases of alcohol droplet are shown in Movie S5. This is also reflected in Fig. 7(b). In Fig. 7(b), we can see that the MCA follows a linear relationship with EA. The same trend holds true for the different liquids used in the experiment. The experimental results are consistent with the theoretical analysis (Fig. 4a). From the experimental results, we also find that the MCA of DI water droplets can reach $180^{\circ}$ with a relatively small EA. And for diethylene glycol droplet, it needs a larger EA, and silicone oil droplet needs an even larger EA. For different liquids, when the EA is fixed, the droplet of higher surface tension will have a larger MCA (Movie S6). When the droplet drops on a smooth and flat surface, namely, the EA is zero, the MCA of the droplet is equal to the ACA. For DI water, diglycol and silicone oil droplets on PDMS substrate, their ACAs are $118 \pm 3^{\circ}, 80 \pm 3^{\circ}$ and $20 \pm 3^{\circ}$, respectively. And the ECAs of these droplets are $108 \pm 3^{\circ}, 75 \pm 3^{\circ}$ and $0 \pm 3^{\circ}$, respectively.

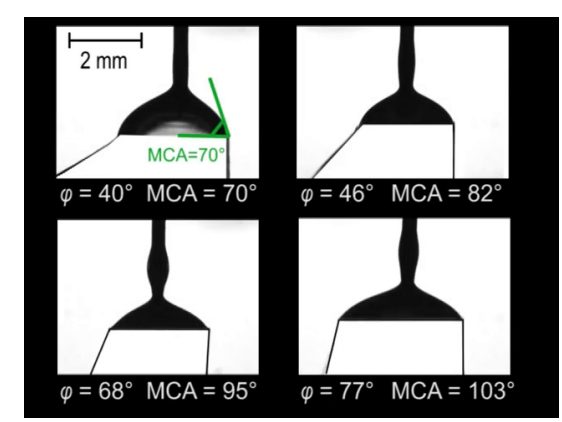

Movie S4. 


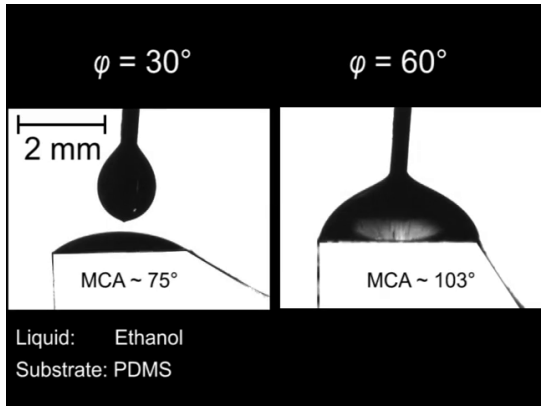

Movie S5.

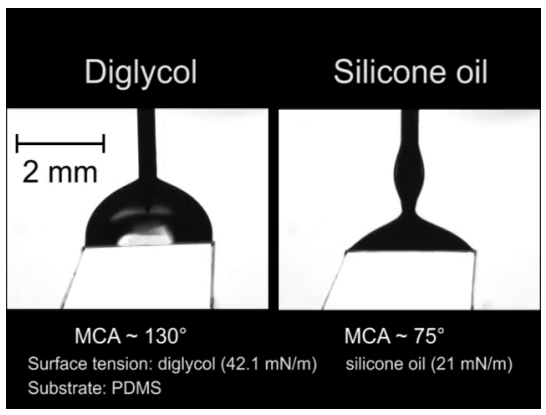

Movie S6.

Fig. 8 illustrates the collapse process of a droplet (Movie S7). Initially, the droplet becomes larger as liquid is continuously injected, and the contact angle increases from the ACA to the MCA. Then, when the contact angle exceeds the MCA, the droplet breaks out from the substrate horizontal surface. And some of liquid, which is originally pinned at the edge, slips to the slope surface. Finally, as the liquids lapse from the horizontal surface, the droplet volume begins to diminish and the contact angle returns to ACA from MCA (Fig. 8a). During this process, the lapsed liquids propagate along the slope surface with a contact angle of ACA. As the liquid lapses continuously, the droplet returns to its initial state where the contact angle is the ACA. The maximum droplet volume that the substrate supports is fixed. In Fig. 8(b), the varia- tion of the droplet contact angle corresponding to Fig. 8(a) is shown. From the experimental data, we can see that the contact angle of a droplet increases initially as the droplet grows, from the ACA to the MCA. After reaching the MCA, the contact angle drops from the MCA to the ACA as the droplet begins to collapse from the substrate. With the continuous injection of liquid into the droplet, the next cycle of contact angle variation begins.

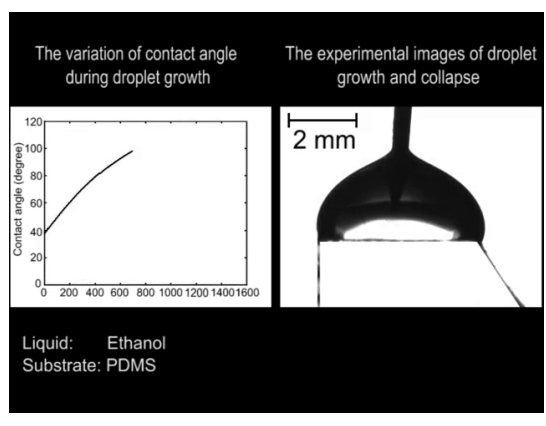

Movie S7.

\subsection{Molecular dynamics simulation of the energy barrier}

Molecular dynamics simulation is adopted to study the energy barrier of one water molecule crossing the substrate edge. The EAs adopted in these simulations are $18.4^{\circ}, 26.6^{\circ}, 45.0^{\circ}, 63.4^{\circ}$, $90^{\circ}, 116.6^{\circ}$ and $135.0^{\circ}$, respectively. The Lennard-Jones (L-J) potential energy, water molecule and substrate made of Au atoms are employed in the simulations. The radius of $\mathrm{Au}$ atom is $134 \mathrm{pm}$, and the surface tension is around $1798 \mathrm{mN} / \mathrm{m}$. Molecular dynamics simulation is carried out in LAMMPS, and the potential energy is the Lennard-Jones (LJ) potential energy:

$E_{i j}=4 \varepsilon_{i j}\left[\left(\frac{\sigma_{i j}}{r_{i j}}\right)^{12}-\left(\frac{\sigma_{i j}}{r_{i j}}\right)^{6}\right]$

where $\varepsilon_{i j}$ is the depth of the potential well, $\sigma_{i j}$ is the zerocrossing distance for the potential, $r_{i j}$ is the distance between the two atoms.
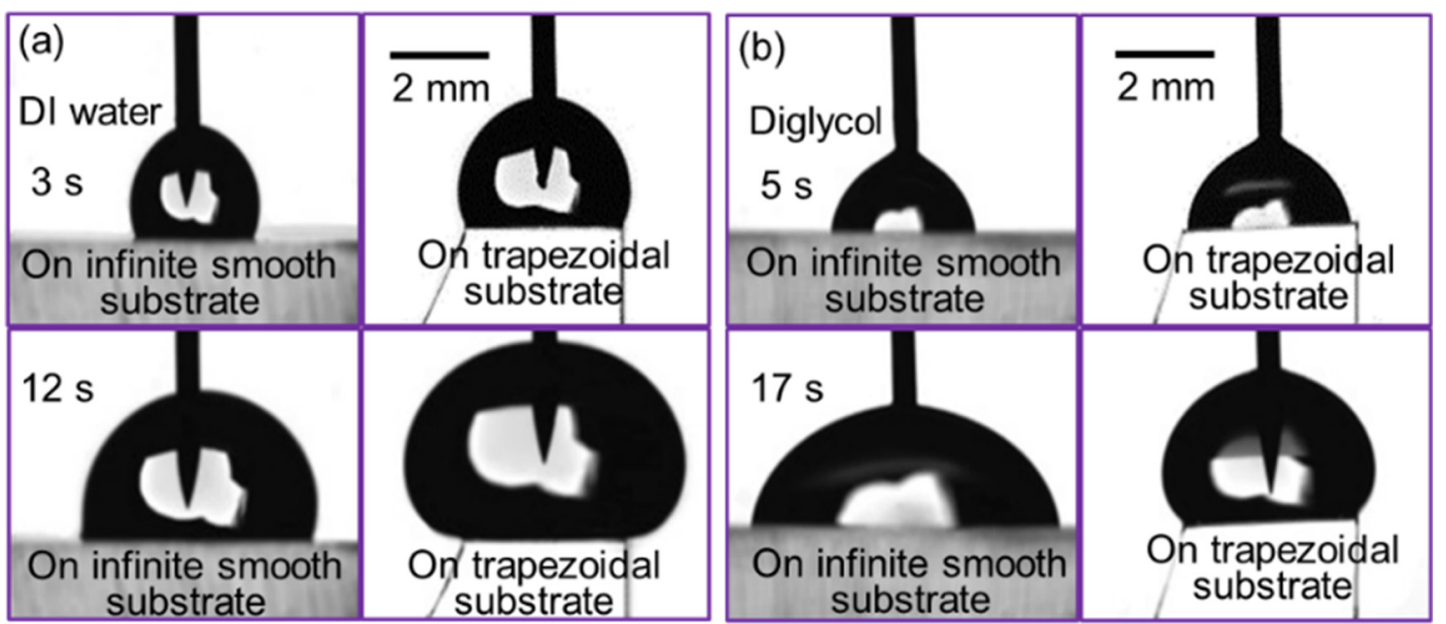

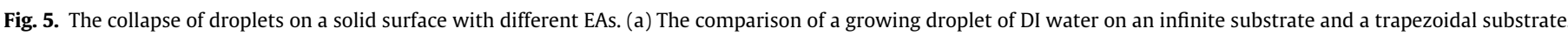

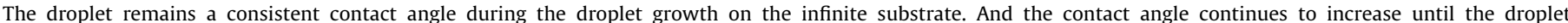

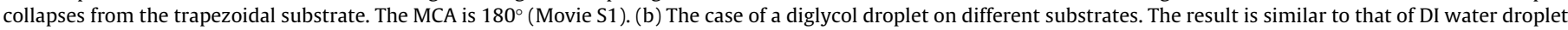
(Movie S2). 


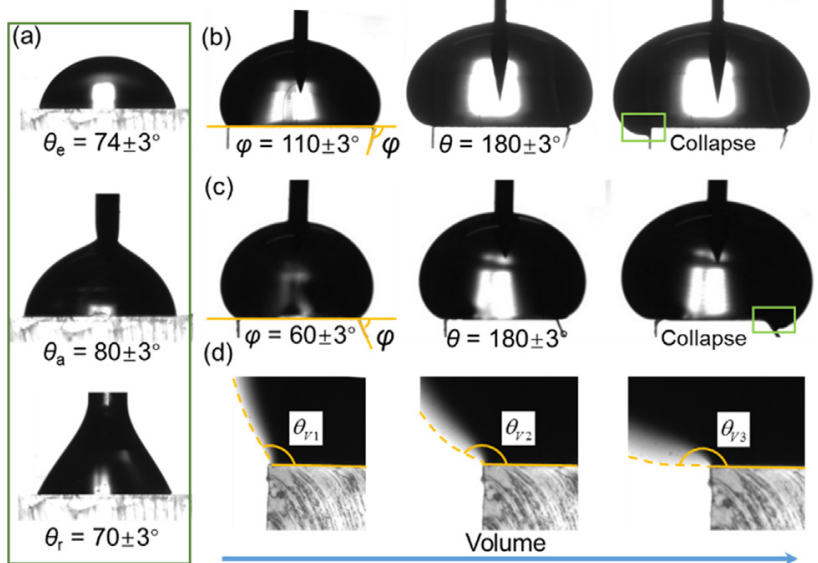

Fig. 6. Droplet contact angles and the collapse from substrate. (a) Diglycol droplet on PDMS surface. $\theta_{\mathrm{e}}$ is of $74 \pm 3^{\circ}$ (upper), $\theta_{\mathrm{a}}$ is $80 \pm 3^{\circ}$ (middle) and $\theta_{\mathrm{r}}$ (receding contact angle) is $70 \pm 3^{\circ}$ (lower). (b) Diglycol droplets on PDMS substrate, with $\varphi$ being $110 \pm 3^{\circ}$. The droplet collapses from the left side of the substrate while the left EA is less than the right EA. (c) Diglycol droplets on PDMS substrate with $\varphi$ being $60 \pm 3^{\circ}$. As the droplet grows, it collapses from the right side of the substrate. (d) The micro view of the pinned section of droplet. As the droplet volume increases, the profile near the contact line flattens, as illustrated by the yellow dashed line. The contact angle approaches $180^{\circ}$ from $\theta_{V 1}$ to $\theta_{V 3}$.

(a)

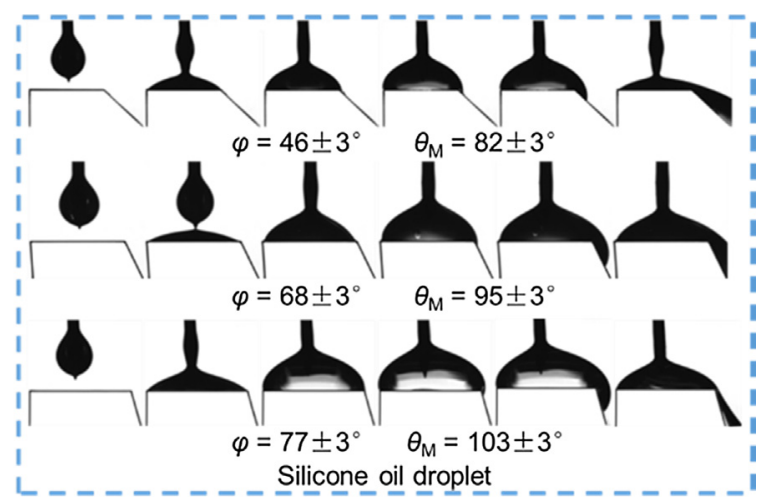

(b)

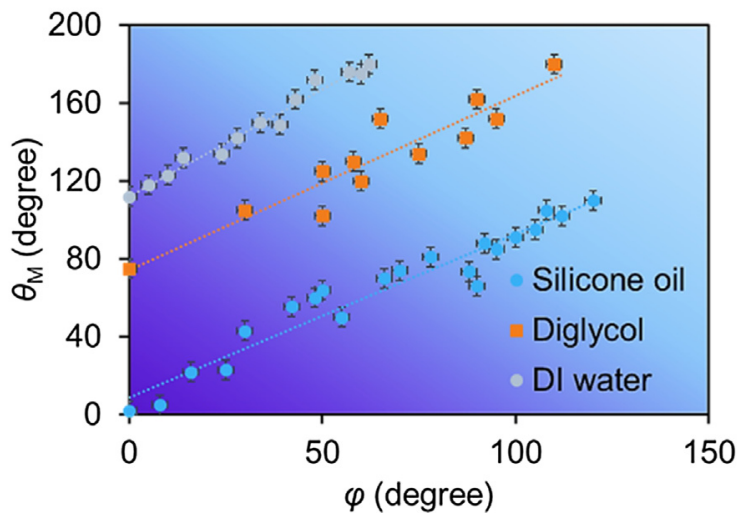

Fig. 7. The relation between MCA and EA. (a) Silicone oil droplet with substrates EA: $\varphi=46 \pm 3^{\circ}, 68 \pm 3^{\circ}, 77 \pm 3^{\circ}$, respectively. The MCAs on these substrates are $82 \pm 3^{\circ}, 95 \pm 3^{\circ}$ and $103 \pm 3^{\circ}$, respectively. The pictures, from left to right, depict the droplets states from growth to collapse. (b) The experimental data comparing the MCA against the EA. The MCA changes with the EA in a linear relationship. This agrees with the theoretical analysis.

The simulation results are shown in Fig. 9. In the simulation, the molecule starts at a distance of $30 \AA$ from the substrate edge, and the distance traveled is denoted as $D$. Fig. 9(a) shows some samples used in the simulation.
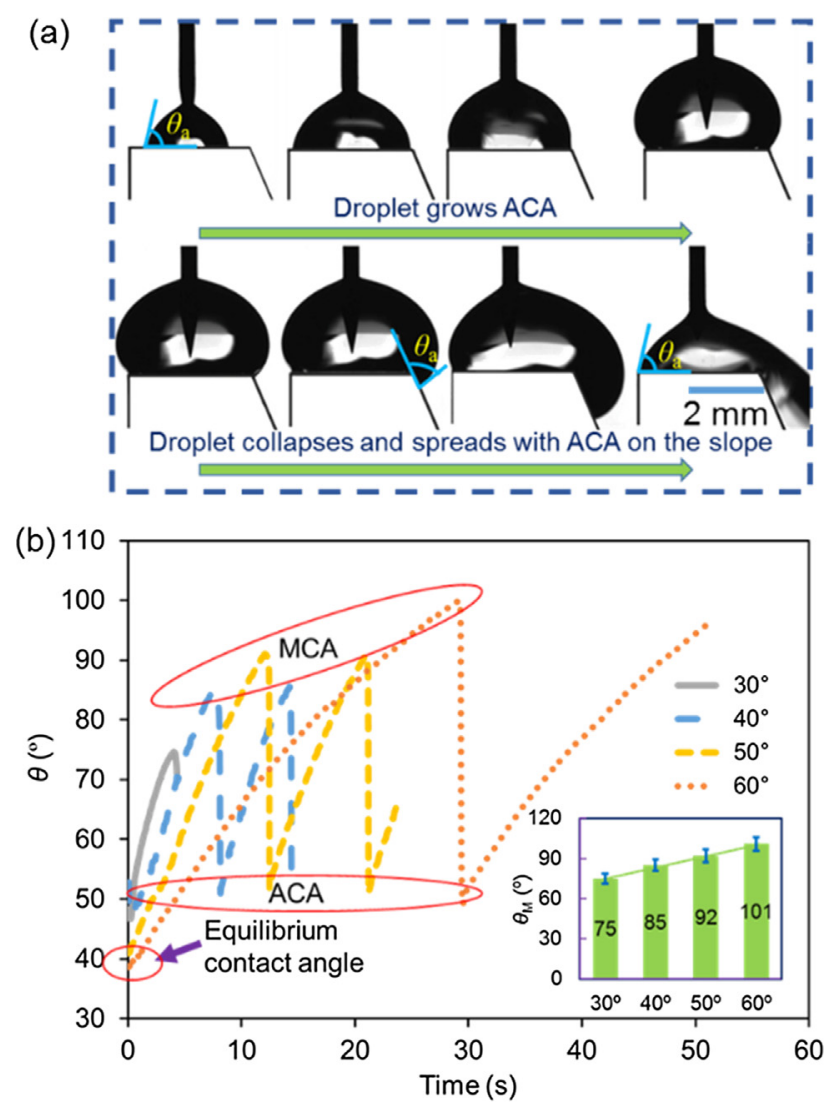

Fig. 8. The variation of contact angle during the process of droplet collapse. (a) The illustration of growth and collapse process of a diglycol droplet on trapezoidal substrate. When the droplet size exceeds the critical volume, the contact line begins to cross the sharp edge, and the front-end liquid spreads to the inclined plane with a contact angle of ACA. This process stops after the contact angle of droplet changes from MCA to ACA. (b) The change of droplet contact angle with time. The injection rate of liquid is $0.025 \underline{\mu l} / \mathrm{s}$. The contact angle increases initially from the ACA to the MCA and then drops to ACA as the droplet collapses. The contact angle starts the next cycle with the continuous injected liquid. The range of the contact angle increases as the EA increases (Movie S7). Insert: the MCAs of droplets on different substrates with different EAs.

Fig. 9(b) shows how the potential energy changes as the molecules move. At the initial distance of $20 \AA$, the molecular potential energy is almost constant and then increases rapidly as the molecule gets closer to the edge. When the molecule crosses the edge, i.e. when $D$ is $30 \AA$, the potential energy reaches its maximum. After that, the potential energy decreases rapidly, and then reverts to the initial value as it moves horizontally (Fig. 9b). From the result, we can see that the sharp edge makes the molecular potential energy increase rapidly. To cross the edge, molecules have to overcome very high energy barriers. For substrates with different EAs, the movement of liquid molecule on the surface shows similar behavior.

The potential energy decreases for most samples with a small EA between $D=10 \AA$ and $D=20 \AA$. The potential energy of water molecule on these samples decreases before $20 \AA$ and increases as $D$ continues to increase. For most samples that had a large EA, the potential energy plateaus as the water molecular transitions from $10 \AA$ to $20 \AA$ (Fig. 9c). In the process of molecular motion, the energy barrier that molecule needs to overcome increases with the increase of EA. This indicates that with increasing EA, it is more difficult for droplet molecules to cross the edge. As a result, the droplets have a larger MCA. The relation between maximum potential energy and EA follows a cosine function, which is consistent with the theoretical analysis results (Fig. 9d). 
(a)
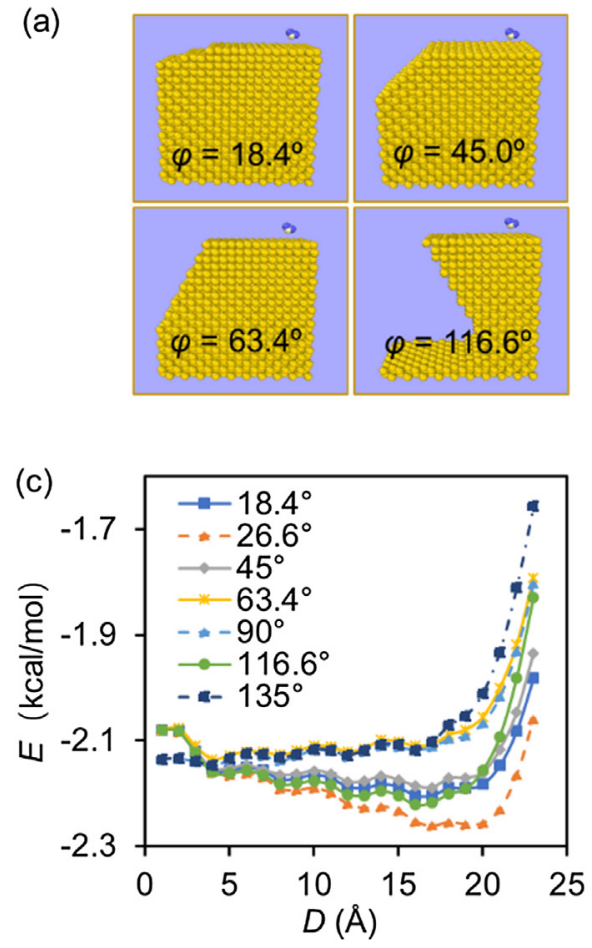
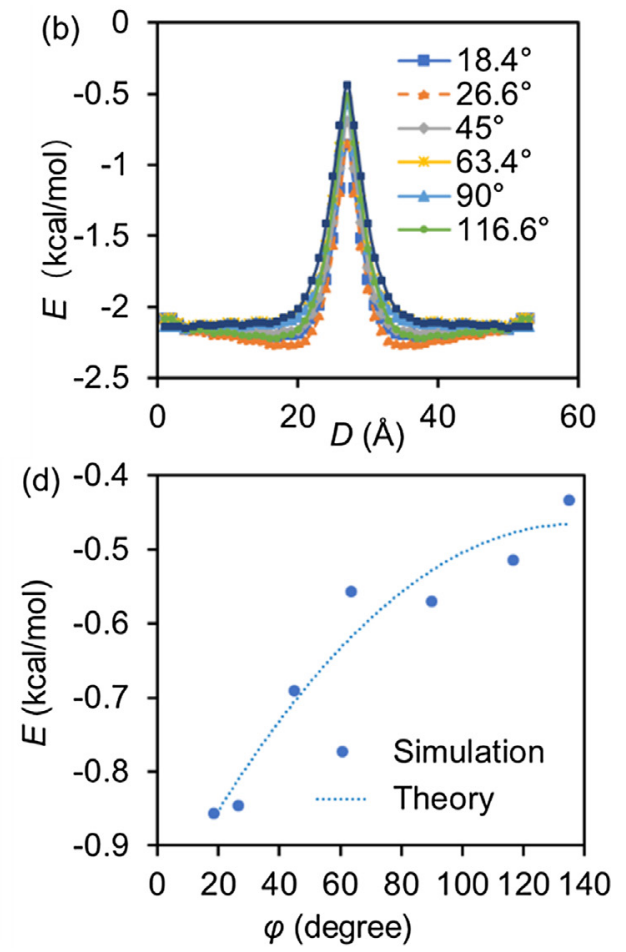

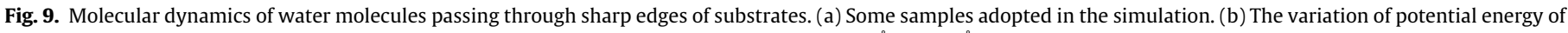

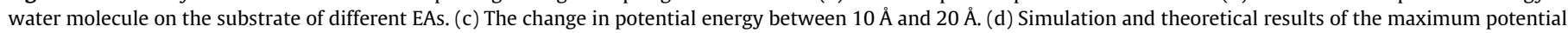
energy of water molecules on the substrates of different EAs, with dots representing simulation results and dotted lines representing theoretical results, respectively.

\subsection{Discussions}

The analysis presented above is rather generic. For surfaces with blunt imperfections, droplets can pass through them relatively easily. In this case, the moving contact line wraps around the relatively blunt defects, and the droplet can easily overcome these obstacles and continue to spread. In this case, surface tension plays a leading role in droplet spreading. In some cases, for example, arrays of micro-pillars surfaces, these defects can even speed up the spreading of droplets. This mainly comes down to the following reasons: (i) the attraction between the liquid and the pillars as the droplet approaches these pillars, (ii) the capillary forces formed between the micro-pillars and the substrate surface during droplet spreading $[27,47]$. We classify these situations as "Contact line Wraps Defects (CWD)". On the other hand, as shown in the experimental results above, the droplet spreading is hindered by the circular connection edge, and the contact line is strongly pinned (Figs. 5 and 7). In this case, the pinning force plays a leading role in the droplet spreading. And the edge can be regarded as a defect of connectivity, which prevents the propagation of liquids significantly. We classify this case as "Defects Wrap Liquids (DWC)." A state between these two cases is also possible. For example, on the surface of arrays of micro-pillars, the degree to which contact lines are pinned depends on the density of micropillars [48-50]. This case was classified as "Mixed Pinning". On the surfaces of plenty of natural and industrial products (e.g., micro channels [51], leaves of plants [52-54] and feather of birds [55]), the second and third states are most common. In these cases, the contact line is difficult to cross the defects, leading to the directional spreading of the droplet along the grooves on channeled surfaces [5], or the immersion of liquids on super-hydrophobic surfaces.

The pinning effect originating from sharp boundaries also provides an effective method for obtaining a fixed volume droplet.
As shown in Fig. 8, the droplet size that can be supported for a particular substrate is constant. When the volume of the droplet exceeds the threshold, the droplet will collapse from the substrate. We can also see this phenomenon via the variation of the contact angle as the volume increases, which is shown in Fig. 9(b). As the droplet grows, the contact angle increases. When the contact angle reaches the maximum, the droplet collapses and the contact angle returns to the initial value. Then, as the liquid continues to be injected into the droplet, the variation of the contact angle recommences. Hence, for a given substrate, liquid and a fixed EA, the droplet size supported by the substrate is fixed.

\section{Conclusions}

In this paper, we prepared the PDMS substrates of different EAs, and carried out the experiments with different liquids whose surface tension changing from large to small. Meanwhile, we carried out relevant theoretical analysis and molecular simulation. The results indicated that the sharp edges have a significant influence on the droplet wetting and dewetting process, which is consistent with the previous results [29-34]. Moreover, our experimental results found that even the totally wetting liquids can have a large contact angle due to the pinning of solid edge, and for more liquids with higher surface tension they can get an apparent contact angle of $180^{\circ}$ on substrates with proper EAs. And this result provides a theoretical basis for the preparation of superhydrophobic surfaces. Experimental and simulation results showed that the sharper the edge, the more firmly the droplet contact line is pinned, and the larger the MCA. The MCA of droplet has a linear relation with EA. Meanwhile, for the first time, we proposed a classification model of pinning effects according to the defects density. The model may cover all cases of droplet contact line pinning. Moving contact line pinning is of great significance for droplet control and 
self-assembly. A large number of studies have been conducted on the close relationship between surface and geometrical morphology of defects, and the establishment of geometrical dependence of pinning force is of great research and application value.

\section{Acknowledgements}

This work was jointly supported by the National Natural Science Foundation of China (NSFC, Grant No. U1562105 and 11372313), and by the Chinese Academy of Sciences (CAS) through CAS Interdisciplinary Innovation Team Project, the CAS Key Research Program of Frontier Sciences (Grant No. QYZDJ-SSWJSC019) and the CAS Strategic Priority Research Program (Grant No. XDB22040401).

\section{References}

[1] T.S. Wong, S.H. Kang, S.K.Y. Tang, E.J. Smythe, B.D. Hatton, A. Grinthal, J Aizenberg, Bioinspired self-repairing slippery surfaces with pressure-stable omniphobicity, Nature 477 (2011) 443-447.

[2] T.W.C. Anish, M.M. Joseph, H.M. Gareth, E.C. Robert, Robust omniphobic surfaces, Proc. Natl. Acad. Sci. USA 105 (2008) 18200-18205.

[3] T.L. Liu, C.J. Kim, Turning a surface superrepellent even to completely wetting liquids, Science 346 (2014) 1096-1100.

[4] P. Calvert, Inkjet printing for materials and devices, Chem. Mater. 13 (2001) 3299-3305.

[5] H. Chen, P. Zhang, H. Liu, Y. Jiang, D. Zhang, Z. Han, L. Jiang, Continuous directional water transport on the peristome surface of nepenthes alata, Nature 532 (2016) 85-89.

[6] A. Tuteja, W. Choi, M. Ma, J.M. Mabry, S.A. Mazzella, G.C. Rutledge, G.H. McKinley, R.E. Cohen, Designing superoleophobic surfaces, Science 318 (2007) $1618-1622$.

[7] A.R. Parker, C.R. Lawrence, Water capture by a desert beetle, Nature 414 (2001) 33-34.

[8] K.C. Park, P. Kim, A. Grinthal, N. He, D. Fox, J.C. Weaver, J. Aizenberg, Condensation on slippery asymmetric bumps, Nature 531 (2016) 78-82.

[9] S. Anand, A.T. Paxson, R. Dhiman, J.D. Smith, K.K. Varanasi, Enhanced condensation on lubricant impregnated nanotextured surfaces, ACS Nano 6 (2012) 10122-10129.

[10] X. Chen, J. Wu, R. Ma, M. Hua, N. Koratkar, S. Yao, Z. Wang, Nanograssed micropyramidal architectures for continuous dropwise condensation, Adv. Funct. Mater. 21 (2011) 4617-4623.

[11] C.H. Chen, Q. Cai, C. Tsai, C.L. Chen, G. Xiong, Y. Yu, Z. Ren, Dropwise condensation on superhydrophobic surfaces with two-tier roughness, Appl Phys. Lett. 90 (2007) 173108.

[12] R.D. Deegan, O. Bakajin, T.F. Dupont, G. Huber, S.R. Nagel, T.A. Witten, Capillary flow as the cause of ring stains from dried liquid drops, Nature 389 (1997) 827-829.

[13] V. Flauraud, D.T.L. Alexander, M. Mastrangeli, E. Shahrabi, G.D. Bernasconi, O.J. F. Martin, J. Butet, J. Brugger, Nanoscale topographical control of capillary assembly of nanoparticles, Nat. Nanotechnol. 12 (2017) 73-80.

[14] J.A. Lv, Y. Liu, J. Wei, E. Chen, L. Qin, Y. Yu, Photocontrol of fluid slugs in liquid crystal polymer microactuators, Nature 537 (2016) 179-184.

[15] N.A. Malvadkar, M.J. Hancock, K. Sekeroglu, W.J. Dressick, M.C. Demirel, An engineered anisotropic nanofilm with unidirectional wetting properties, Nat. Mater. 9 (2010) 1023-1028.

[16] A.K. Kota, Y. Li, J.M. Mabry, A. Tuteja, Hierarchically structured superoleophobic surfaces with ultralow contact angle hysteresis, Adv. Mater 24 (2012) 5838-5843.

[17] S. Herminghaus, M. Brinkmann, R. Seemann, Wetting and dewetting of complex surface geometries, Annu. Rev. Mater. Res. 38 (2008) 101-121.

[18] D. Quéré, Wetting and roughness, Annu. Rev. Mater. Res. 38 (2008) 71-99.

[19] D. Mannetje, S. Ghosh, R. Lagraauw, S. Otten, A. Pit, C. Berendsen, J. Zeegers, D. van den Ende, F. Mugele, Trapping of drops by wetting defects, Nat. Commun. 5 (2014) 3559-3565.

[20] U. Thiele, Patterned deposition at moving contact lines, Adv. Colloid Interfac. 206 (2014) 399-413.

[21] E. Adachi, A.S. Dimitrov, K. Nagayama, Stripe patterns formed on a glass surface during droplet evaporation, Langmuir 11 (1995) 1057-1060.

[22] H. Bodiguel, F. Doumenc, B. Guerrier, Stick-slip patterning at low capillary numbers for an evaporating colloidal suspension, Langmuir 26 (2010) 1075810763.
[23] G.D. Nadkarni, S. Garoff, An investigation of microscopic aspects of contact angle hysteresis pinning of the contact line on a single defect, EPL 20 (1992) 523-528.

[24] P.G. de Gennes, Wetting states and dynimacs, Rev. Mod. Phys. 57 (1985) 827 863.

[25] Y.P. Zhao, Physical Mechanics of Surfaces and Interfaces, Science Press, Beijing, 2012.

[26] T. Young, An essay on the cohesion of fluids, Phil. Trans. Roy. Soc. 95 (1805) 65-87.

[27] P. Papadopoulos, L. Mammen, X. Deng, D. Vollmer, H.J. Butt, How superhydrophobicity breaks down, Proc. Natl. Acad. Sci. USA 110 (2013) $3254-3258$.

[28] E. Bormashenko, Progress in understanding wetting transitions on rough surfaces, Adv. Colloid Interfac. 222 (2015) 92-103.

[29] J.F. Oliver, C. Huh, S.G. Mason, Resistance to spreading of liquids by sharp edges, J. Colloid Interf. Sci. 59 (1977) 568-581.

[30] R. Hensel, R. Helbig, S. Aland, H.G. Braun, A. Voigt, C. Neinhuis, C. Werner, Wetting resistance at its topographical limit: the benefit of mushroom and serif T structures, Langmuir 29 (2013) 1100-1112.

[31] W.J.A. de Wijs, J. Laven, G. de With, Wetting forces and meniscus pinning at geometrical edges, AIChE J. 62 (2016) 4453-4465.

[32] O.J. Romero, L.E. Scriven, M.D.S. Carvalho, Effect of curvature of coating die edges on the pinning of contact line, AIChE J. 52 (2006) 447-455.

[33] V. Grishaev, A. Amirfazli, S. Chikov, Y. Lyulin, O. Kabov, Study of edge effect to stop liquid spillage for microgravity application, Microgravity Sci. Technol. 25 (2012) 27-33.

[34] G. Fang, A. Amirfazli, Understanding the edge effect in wetting: a thermodynamic approach, Langmuir 28 (2012) 9421-9430.

[35] F.C. Wang, H.A. Wu, Pinning and depinning mechanism of the contact line during evaporation of nano-droplets sessile on textured surfaces, Soft Matter 9 (2013) 5703-5709.

[36] A. Giacomello, L. Schimmele, S. Dietrich, Wetting hysteresis induced by nanodefects, Proc. Natl. Acad. Sci. USA 113 (2016) E262-E271.

[37] C.U. Chan, L. Chen, M. Arora, C.D. Ohl, Collapse of surface nanobubbles, Phys. Rev. Lett. 114 (2015) 114505.

[38] R. Fetzer, J. Ralston, Exploring defect height and angle on asymmetric contact line Pinning, J. Phys. Chem. C 115 (2011) 14907-14913.

[39] Y.V. Kalinin, V. Berejnov, R.E. Thorne, Contact line pinning by microfabricated patterns: effects of microscale topography, Langmuir 25 (2009) 5391-5397.

[40] H. Perrin, R. Lhermerout, K. Davitt, E. Rolley, B. Andreotti, Defects at the nanoscale impact contact line motion at all scales, Phys. Rev. Lett. 116 (2016) 184502.

[41] W. Xu, C.H. Choi, From sticky to slippery droplets: dynamics of contact line depinning on superhydrophobic surfaces, Phys. Rev. Lett. 109 (2012) 024504.

[42] J. Li, D. Han, Y.P. Zhao, Kinetic behavior of the cells touching substrate: the interfacial stiffness guides cell spreading, Sci. Rep. 4 (2014) 3910.

[43] L. Limat, Straight contact lines on a soft, incompressible solid, Eur. Phys. J. E. Soft Matter 35 (2012) 9811.

[44] T. Ondarcuhu, A. Piednoir, Pinning of a contact line on nanometric steps during the dewetting of a terraced substrate, Nano Lett. 5 (2005) 1744-1750.

[45] R.J. Good, M.N. Koo, The effect of drop size on contact angle, J. Colloid Interf. Sci. 71 (1979) 283-292.

[46] M.V. Bartashevich, V.V. Kuznetsov, O.A. Kabov, Gravity effect on the axisymmetric drop spreading, Microgravity Sci. Technol. 22 (2010) 107-114.

[47] Q. Yuan, X. Huang, Y. Zhao, Dynamic spreading on pillar-arrayed surfaces: viscous resistance versus molecular friction, Phys. Fluids 26 (2014) 092104.

[48] P.S. Forsberg, C. Priest, M. Brinkmann, R. Sedev, J. Ralston, Contact line pinning on microstructured surfaces for liquids in the Wenzel state, Langmuir 26 (2010) 860-865.

[49] D. Bonn, J. Eggers, J. Indekeu, J. Meunier, E. Rolley, Wetting and spreading, Rev. Mod. Phys. 81 (2009) 739-805.

[50] T. Cubaud, M. Fermigier, Advancing contact lines on chemically patterned surfaces, J. Colloid Interf. Sci. 269 (2004) 171-177.

[51] Z. Wang, Y.P. Zhao, Wetting and electrowetting on corrugated substrates, Phys. Fluids 29 (2017) 067101

[52] A. Roth-Nebelsick, M. Ebner, T. Miranda, V. Gottschalk, D. Voigt, S. Gorb, T. Stegmaier, J. Sarsour, M. Linke, W. Konrad, Leaf surface structures enable the endemic namib desert grass stipagrostis sabulicola to irrigate itself with fog water, J. R. Soc. Interface 9 (2012) 1965-1974.

[53] K. Liu, X. Yao, L. Jiang, Recent developments in bio-inspired special wettability, Chem. Soc. Rev. 39 (2010) 3240-3255.

[54] D. Wu, J.N. Wang, S.Z. Wu, Q.D. Chen, S. Zhao, H. Zhang, H.B. Sun, L. Jiang, Three-level biomimetic rice-leaf surfaces with controllable anisotropic sliding, Adv. Funct. Mater. 21 (2011) 2927-2932.

[55] M. Prakash, D. Quéré, J.W. Bush, Surface tension transport of prey by feeding shorebirds: the capillary ratchet, Science 320 (2008) 931-934. 